\title{
Effet de vent latéral sur un modèle simplifié de voiture par une méthode DES
}

\author{
Emmanuel Guilmineau ${ }^{a}$, Oussama Chikhaoui, Ganbo Deng et Michel Visonneau \\ Laboratoire de Mécanique des Fluides, CNRS UMR 6598, École Centrale de Nantes, B.P. 92101, 44321 Nantes Cedex 3, France
}

Reçu le 20 April 2009

\begin{abstract}
Résumé - Cette étude traite des effets du vent latéral sur un modèle simplifié de voiture, appelé le modèle de Willy. Ce modèle sans arête sur la partie avant et avec un culot droit est plus adapté pour l'analyse des séparations instationnaires qui sont limitées au côté sous le vent et au culot. Les simulations sont réalisées à l'aide du code de calcul ISIS-CFD par une approche DES et les résultats sont comparés à des données expérimentales ainsi qu'à des résultats obtenus par une simulation RANSE. Les comparaisons portent sur la pression pariétale, la pression totale ainsi que sur les efforts et moment de lacet.
\end{abstract}

Mots clés : Vent latéral / aérodynamique / DES / modélisation de la turbulence

Abstract - Effects of crosswind on a simplified car model with a DES method. This study deals with the crosswind effects on a simplified car model, named the Willy model. This model without sharp corner on the fore body and a square base is more convenient for the analysis of unsteady separations limited on its leeward side and base. The simulations are carried out with the flow solver ISIS-CFD by a DES approach and the results are compared to the experimental data and to the results obtained by a RANSE simulation. The comparisons deal with pressure, total pressure and forces and yawing moment.

Key words: Crosswind / aerodynamics / DES / turbulence

\section{Introduction}

Comme les véhicules automobiles deviennent plus légers pour réduire leur consommation de carburant, la stabilité devient plus sensible aux forces aérodynamiques. De plus, un véhicule sur la route est soumis à un écoulement atmosphérique turbulent et non uniforme ainsi qu'à des vents latéraux transitoires. Ces phénomènes, tels que le dépassement ou le croisement, sont des facteurs à prendre en compte pour la stabilité dynamique du véhicule [1].

La simulation numérique est bien intégrée dans l'industrie automobile et est maintenant un outil ingénieur utilisé en parallèle avec l'expérience durant la phase de design des véhicules. D'un point de vue recherche, le corps de Ahmed est le modèle le plus couramment choisi. Mais à ce jour, la plupart des simulations numériques des effets du vent latéral sur des véhicules terrestres sont réalisées sur des camions [2] et des trains [3]. Pour les automobiles, l'étude du vent latéral par des expériences a déjà été réalisé $[4,5]$. Par contre, d'un point de vue numérique, ceci est beaucoup plus récent [6].

\footnotetext{
a Auteur pour correspondance :

emmanuel.guilmineau@ec-nantes.fr
}

Le but de cet article est d'utiliser une simulation de la turbulence par une approche DES pour étudier un écoulement décollé autour d'un corps massif avec l'application d'un vent latéral sur ce corps. Le modèle simplifié est le corps de Willy [7] qui est un corps ne présentant pas d'arêtes. Un modèle générique sans coins sur tout l'avant du corps et un culot droit est plus adapté pour analyser les séparations qui sont limitées au côté et au culot. Une alternative aurait été d'employer le corps de Ahmed qui est construit autour d'une boite rectangulaire. Ce modèle est très utilisé pour analyser les contributions de chaque partie du modèle dans la traînée et sert couramment comme corps basique pour des comparaisons CFD. Avec un vent latéral, ce modèle présenterait de larges décollements au niveau de l'intersection du côté avec le toit qui interagiraient avec les vortex générés par la lunette arrière et qui rendraient difficile l'analyse du vent latéral. Pour ces raisons, le modèle de Ahmed n'a pas été retenu.

\section{Modèle de Willy}

Le modèle de Willy est un modèle avec un culot droit, qui est réaliste comparé à un véhicule de type monospace. 


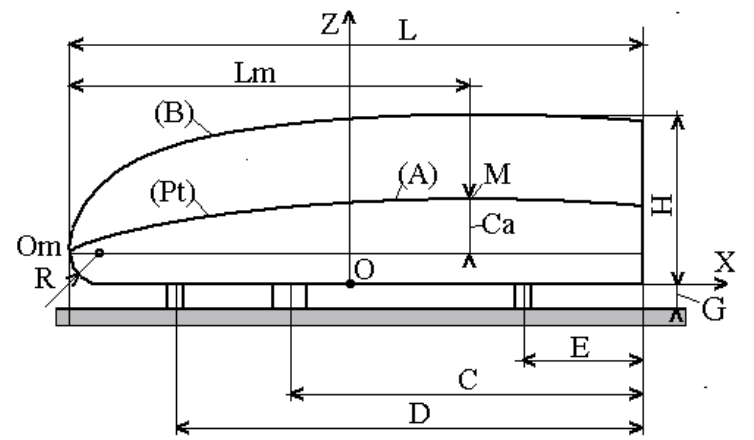

(a)

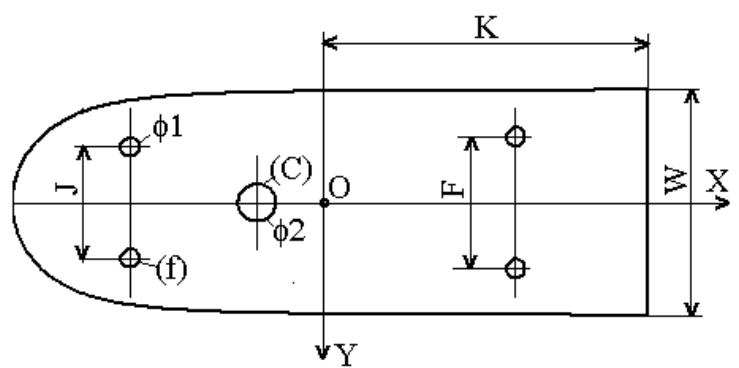

(b)

Fig. 1. Définition du modèle de Willy, (a) vue de côté, (b) vue de dessous.

Ce modèle est utilisé pour la première fois par Chometon et al. [7]. Le modèle est présenté sur les figures 1. La longueur du modèle est $L=675 \mathrm{~mm}$, la largeur $240 \mathrm{~mm}$, la hauteur maximale $192 \mathrm{~mm}$ et la surface de référence est Sref $=41791 \mathrm{~mm}^{2}$. La garde au sol du modèle est $G=29 \mathrm{~mm}$ et le diamètre des quatre pieds (f) qui soutiennent le modèle est $\phi_{1}=20 \mathrm{~mm}$. Un cylindre (c) de diamètre $\phi_{2}=40 \mathrm{~mm}$ est utilisé pour protéger le faisceau de câbles qui sert pour les mesures de pression. Les autres dimensions sont définies dans [8]. Le référentiel $(X o, Y o, Z o)$ est le référentiel Eiffel où l'axe $X o$ est parallèle à la vitesse amont. Le référentiel $(X, Y, Z)$ est lié au modèle. L'origine de ces référentiels est liée au point O situé au plancher du modèle et à $330 \mathrm{~mm}$ de l'avant du modèle, voir figure 1 . Ce point $\mathrm{O}$ est le centre de rotation $\mathrm{du}$ modèle.

La vitesse amont de l'écoulement est $V_{0}=20 \mathrm{~m} / \mathrm{s}$, soit un nombre de Reynolds, basé sur la longueur du corps, $R e=0,9 \times 10^{6}$. L'angle de dérapage $\beta$ est positif si le côté droit du modèle est le côté au vent.

\section{Approche numérique}

\subsection{Le solveur Navier-Stokes}

Le code de calcul ISIS-CFD, développé par l'Équipe Modélisation Numérique du Laboratoire de Mécanique des Fluides de l'École Centrale de Nantes, utilise les équations instationnaires de Navier-Stokes en moyenne de Reynolds pour simuler un fluide visqueux et incompressible. Le solveur est basé sur une méthode volume fini pour construire une discrétisation spatiale des équations de transport. La méthode basée sur la face est généralisée à des maillages bidimensionnels ou tridimensionnels nonstructurés pour lesquels les volumes de contrôle sont composés d'un nombre arbitraire de faces.

Le champ de vitesse est obtenu à partir des équations de conservation de quantité de mouvement et le champ de pression est déduit de la contrainte de conservation de la masse, ou équation de continuité, transformée en une équation de pression. Les intégrales surfaciques et volumiques sont évaluées en utilisant une approximation du second ordre [9].
Dans cette étude, le modèle DES-SST présenté par Menter et Kuntz [10] est le modèle de turbulence choisi.

Dans cette approche, la procédure appliquée par Travin et al. [11] pour définir la transition RANS/LES est utilisée dans le modèle $k$ - $\omega$ SST de Menter [12]. Dans ce cas, le terme de destruction, qui est égal à $E=\beta \omega k$, devient en DES $E=\beta \omega k F_{\text {DES }}$ où la fonction $F_{\text {DES }}$ est définie par :

$$
F_{\mathrm{DES}}=\max \left(\frac{L_{\mathrm{t}}}{C_{\mathrm{DES}} \Delta}, 1\right)
$$

avec $L_{\mathrm{t}}$, la longueur turbulente naturelle définie par $L_{\mathrm{t}}=$ $\sqrt{k} /(\beta \omega)$.

Le pas de temps utilisé dans les simulations DES est $\Delta t=0,0003 \mathrm{~s}$, soit $110 \Delta t$ par temps de référence $\left(T_{\text {ref }}=\right.$ $\left.L / V_{0}\right)$.

\subsection{Maillage et conditions aux limites}

Le domaine de calcul commence à $4,4 \times L$ de l'origine $\mathrm{O}$ devant le modèle et s'étend à $5,9 \times L$ derrière le modèle. La largeur du domaine est $3 \times L$ et la hauteur $1,6 \times L$. Le maillage est généré en utilisant HEXPRESSTM ${ }^{\mathrm{TM}}$, un mailleur non-structuré automatique. Ce mailleur génère des maillages contenant uniquement des hexaèdres. Le maillage est composé d'environ 6,6 millions de points avec approximativement 103000 points pour décrire le modèle et 50000 pour le sol de la soufflerie. Pour la surface du modèle, une condition de non-glissement est choisie et la résolution de la distance normale à la paroi est de 0,006 mm. Le sol du tunnel de la soufflerie est vu par une fonction de paroi et la distance entre le sol et les premiers points du domaine fluide est de $0,6 \mathrm{~mm}$. Cette approche, utilisée pour prendre en compte les parois, est la même que celle proposée par Krajnovic et Davidson [13]. A la sortie du domaine une condition de pression imposée est utilisée alors que les autres frontières possèdent une condition de Dirichlet.

\section{Résultats}

Les résultats obtenus par les simulations DES sont comparés par rapport à des données expérimentales ainsi 


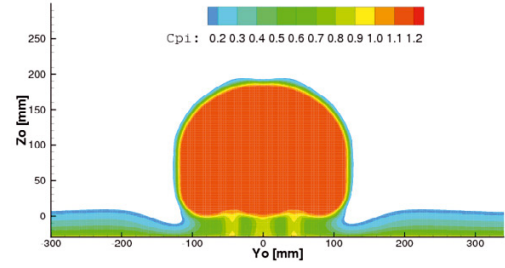

(a)

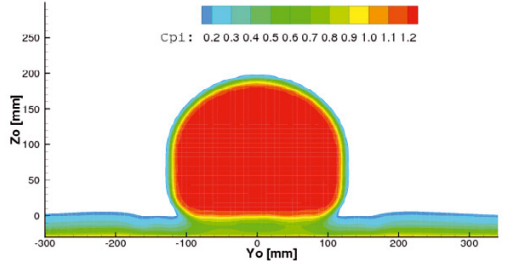

(b)

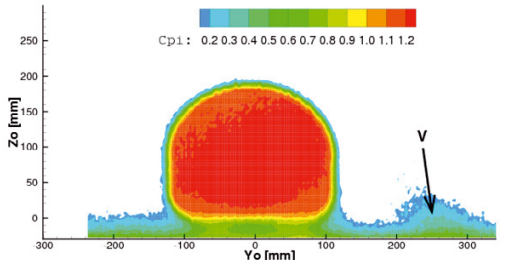

(c)

Fig. 2. Coefficient de pression totale à $X_{o} / L=0,60$ pour $\beta=0^{\circ}$, (a) EASM, (b) DES, (c) expérience.

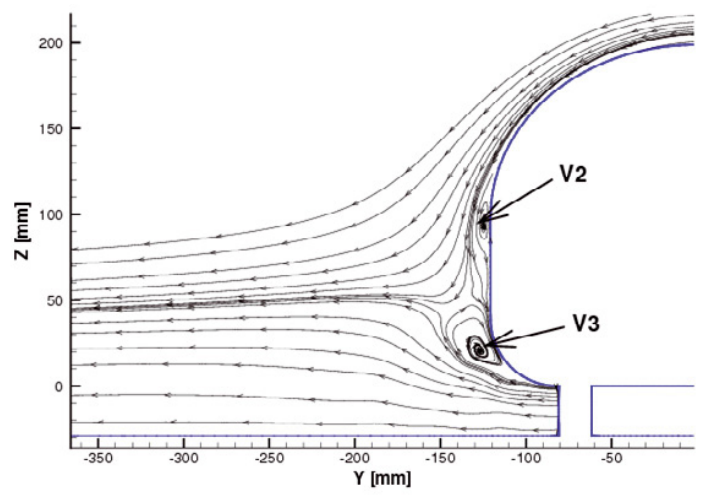

(a)

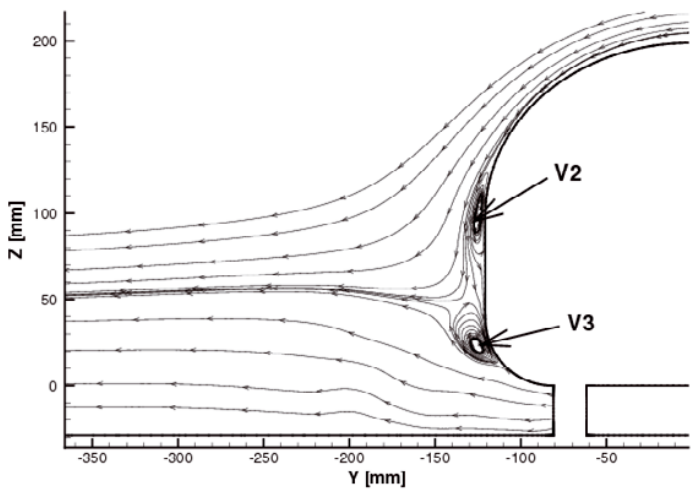

(b)

Fig. 3. Écoulement secondaire à $X / L=0,30$ pour $\beta=10^{\circ}$, (a) EASM, (b) DES.

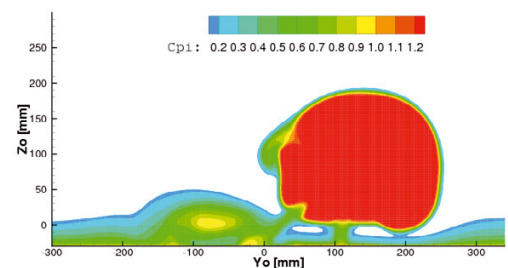

(a)

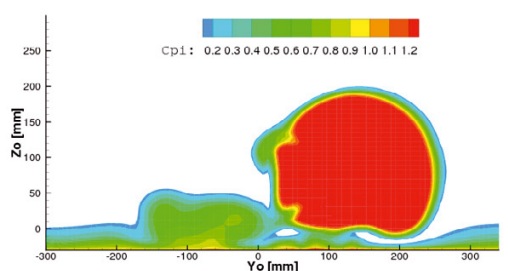

(b)

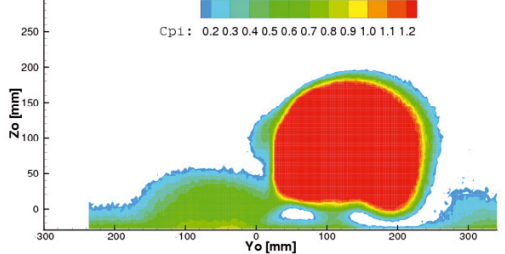

(c)

Fig. 4. Coefficient de pression totale à $X_{o} / L=0,60$ pour $\beta=20^{\circ}$, (a) EASM, (b) DES, (c) expérience.

qu'à des résultats obtenus avec le modèle de turbulence non-linéaire EASM [8].

\subsection{Structure de l'écoulement}

Les figures 2, 4, 6 représentent le coefficient de pression totale dans un plan perpendiculaire à la vitesse amont à l'abscisse $X_{o} / L=0,60$ pour les angles de dérapage de $0^{\circ}$ et $20^{\circ}$ et à l'abscisse $X_{o} / L=0,55$ pour l'angle de dérapage $\beta=30^{\circ}$. Pour l'angle $\beta=0^{\circ}$, voir figure 2 , on note que l'écoulement reste attaché. Seule, une bulle de recirculation apparaît dans le sillage. Sa longueur dépend de la modélisation de la turbulence choisie. Avec le modèle EASM, la longueur est de $0,50 \times L$ alors qu'avec la simulation DES, la longueur est de $0,44 \times L$. Dans une autre expérience [14] où le cylindre (c) n'est pas présent, la longueur de cette bulle de recirculation mesure $0,4 \times L$. Dans les expériences, voir figure $2 c$, une accumulation de pression totale, notée $V$, est due à l'interaction d'un obstacle amont avec la couche limite du sol de la soufflerie. Quand l'angle de dérapage augmente, la couche limite épaissit jusqu'à produire une zone de décollement, notée $V 2$, comme le montre la figure 3 pour l'incidence $\beta=10^{\circ}$. Ce vortex $V 2$ est plus étendu dans les résultats obtenus par une simulation DES, voir figure $3 \mathrm{~b}$, que dans les résultats obtenus avec le modèle EASM. On constate également un deuxième vortex, situé sous le vortex $V 2$, noté $V 3$ situé vers le bas du modèle, qui est attaché au modèle. Plus l'angle de dérapage augmente, plus la taille du vortex $V 2$ augmente.

Pour l'incidence $\beta=20^{\circ}$, la taille du décollement $V 2$ sur le côté sous le vent a augmenté. On observe également une zone d'augmentation de la pression totale centrée vers $Y o=-100 \mathrm{~mm}$. On note également que quand l'angle de dérapage augmente, la position du vortex $V 3$ dépend de la modélisation de la turbulence. Avec un modèle nonlinéaire, voir figure $5 \mathrm{a}$, ce vortex est déjà complètement 


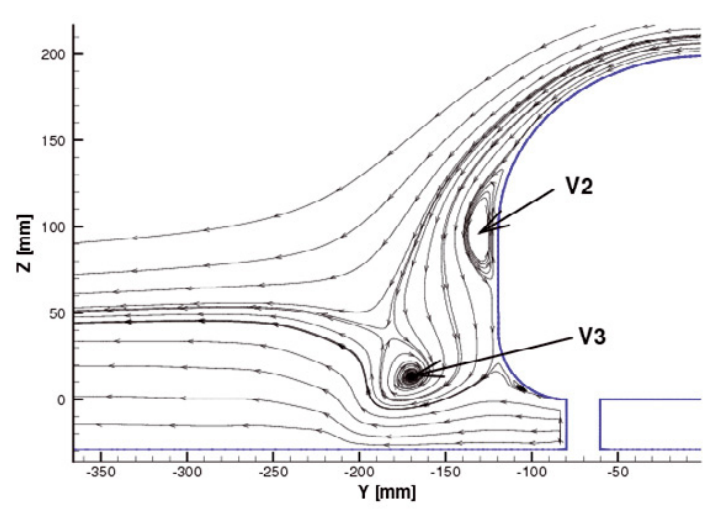

(a)

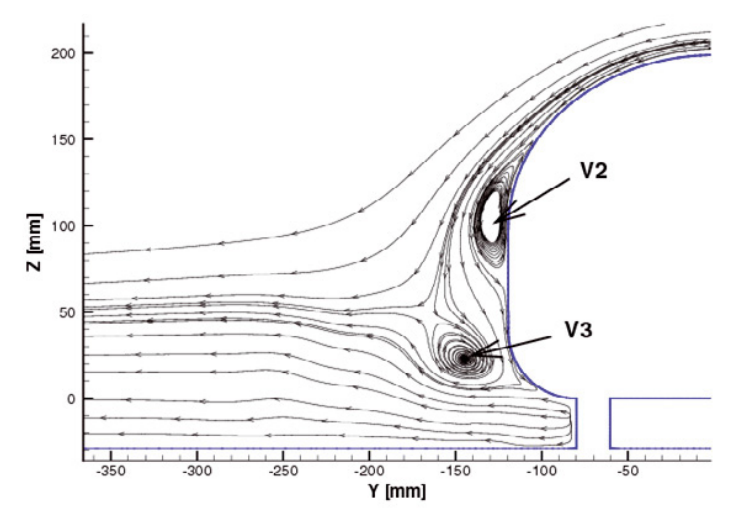

(b)

Fig. 5. Écoulement secondaire à $X / L=0,30$ pour $\beta=20^{\circ}$, (a) EASM, (b) DES.

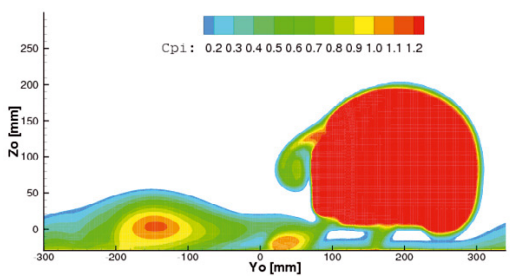

(a)

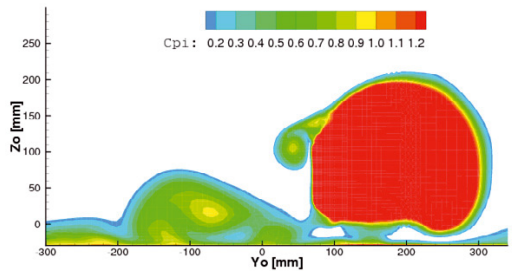

(b)

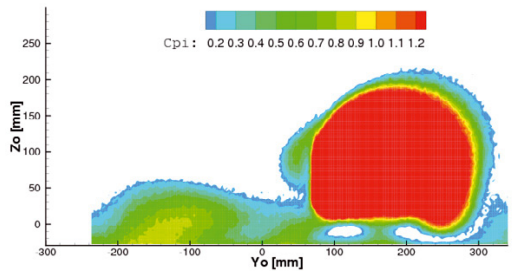

(c)

Fig. 6. Coefficient de pression totale à $X_{o} / L=0,55$ pour $\beta=30^{\circ}$, (a) EASM, (b) DES, (c) expérience.

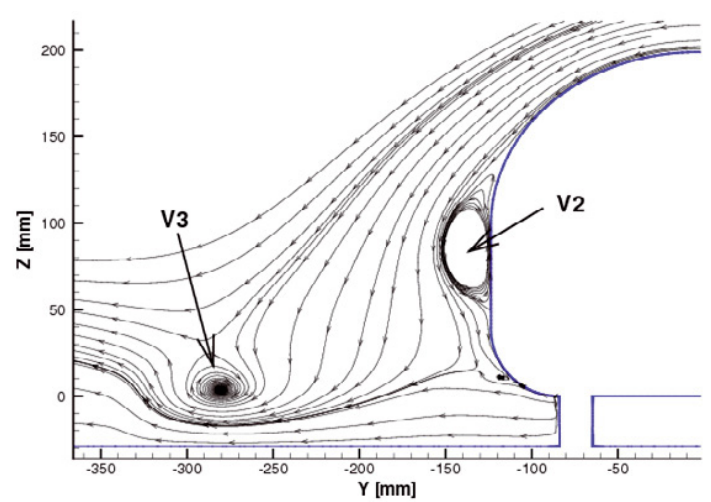

(a)

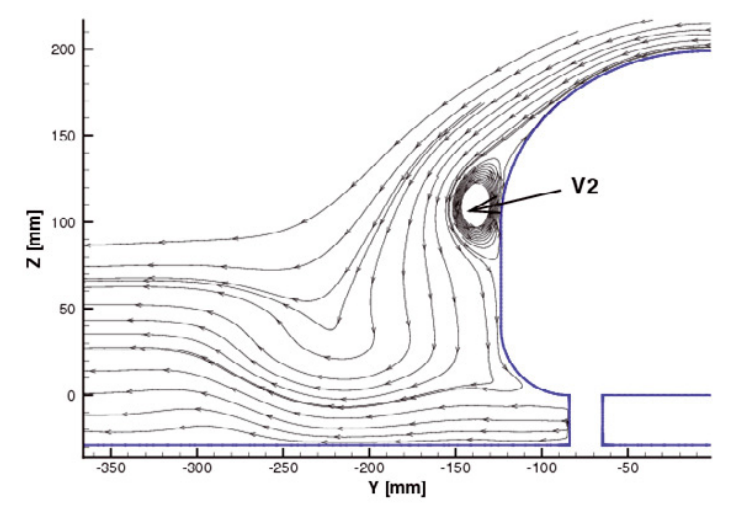

(b)

Fig. 7. Écoulement secondaire à $X / L=0,30$ pour $\beta=30^{\circ}$, (a) EASM, (b) DES.

séparé du corps, ce qui n'est pas le cas avec la simulation DES.

Pour l'angle de dérapage $\beta=30^{\circ}$, la position du vortex $V 2$ et la taille sont différentes entre les deux modélisations de la turbulence, ce qui est clairement visible sur les figures 6 et 7 . On constate que le vortex $V 3$ est très éloigné du corps avec la modélisation EASM alors que dans la simulation DES, celui-ci est diffusé. La zone située entre $Y_{o}=-300 \mathrm{~mm}$ et $Y_{o}=-200 \mathrm{~mm}$ correspond au vortex $V 3$, qui est issu de l'interaction des sillages des pieds avant du modèle avec le sillage du cylindre (c), comme le montrent les figures 8 . On constate que le sillage des pieds est plus intense dans les résultats obtenus avec le modèle EASM que dans les résultats obtenus dans les simulations DES. Ceci est vrai quel que soit l'angle de dérapage $\beta$.

\subsection{Pression pariétale et forces}

L'évolution du coefficient de pression $C_{\mathrm{p}}$ le long de la courbe $(\mathrm{Pt})$, qui est une projection de la courbe (A) sur les côtés du modèle, est présentée sur les figures 9 pour différents angles de dérapage $\beta$. On note que le point de 


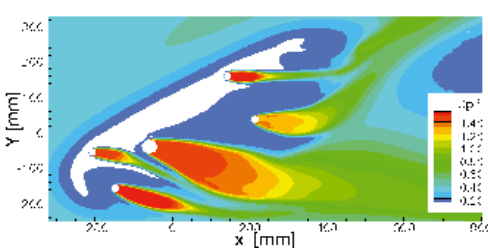

(a)

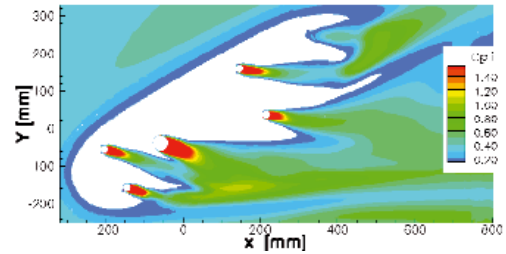

(b)

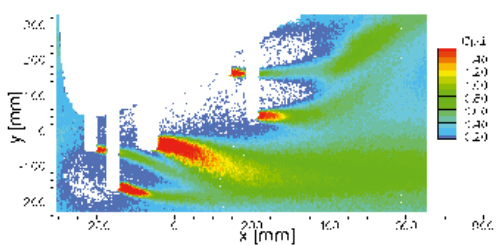

(c)

Fig. 8. Coefficient de pression totale à $Z_{o}=-14,5 \mathrm{~mm}$ pour $\beta=30^{\circ}$, (a) EASM, (b) DES, (c) expérience.

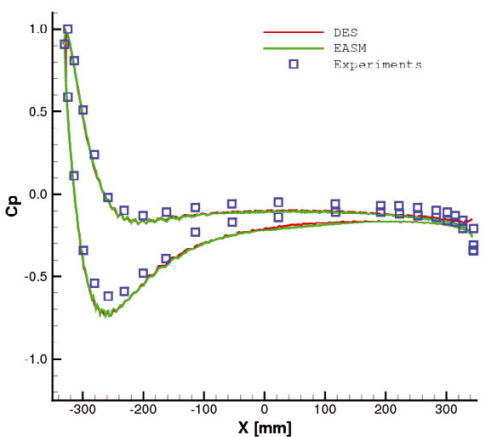

(a)

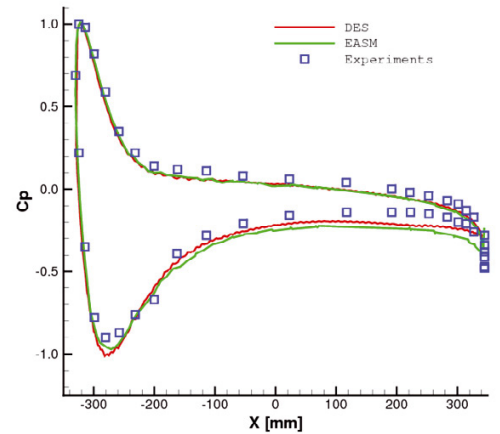

(b)

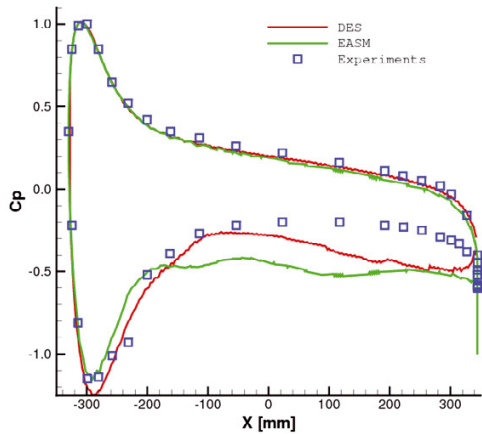

(c)

Fig. 9. Coefficient de pression sur la courbe (Pt), (a) $\beta=10^{\circ}$, (b) $\beta=20^{\circ}$, (c) $\beta=30^{\circ}$.

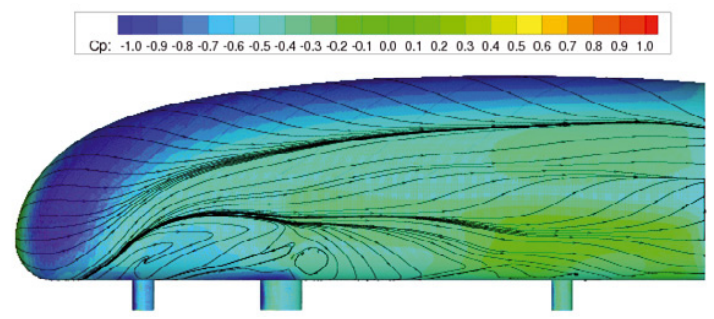

(a)

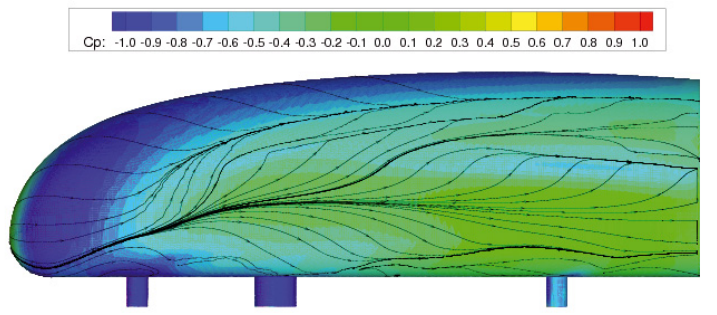

(b)

Fig. 10. Coefficient de pression sur le côté sous le vent pour $\beta=30^{\circ}$, (a) EASM, (b) DES.

stagnation est situé sur le côté au vent et que celui-ci recule quand l'angle de dérapage augmente. Les résultats montrent que la pression à la paroi est correctement simulée par les deux modélisations de la turbulence pour le côté au vent pour toutes les incidences. Pour le côté sous le vent, l'écoulement se sépare quand l'angle de dérapage est supérieur à $10^{\circ}$. Le gradient de pression négatif augmente et l'accord entre les calculs et les expériences est correct jusqu'au changement de signe du gradient de pression. A $X=-200 \mathrm{~mm}$, approximativement, le changement de pression est une indication du début de la zone de décollement pour les résultats obtenus avec la modélisation EASM. Ceci est confirmé par les figures 10 qui donnent une cartographie de la pression pour l'angle de dérapage $\beta=30^{\circ}$. On note que la zone de basse pression s'étend jusqu'au haut du côté au vent du modèle. On remarque également que le sillage du cylindre central (c) perturbe plus l'écoulement dans les simulations avec le modèle EASM que dans les simulations DES.
Le coefficient de pression est également tracé sur le culot du modèle à $Z=50 \mathrm{~mm}$ pour plusieurs angles de dérapage $\beta$ sur les figures 11 . On peut noter que la pression obtenue avec la modélisation EASM est presque constante sur tout le culot, excepté pour l'angle $\beta=30^{\circ}$ où le maximum de pression se situe au milieu du culot. Or dans les expériences, la pression est maximale vers le bord du culot situé au côté au vent. Les simulations DES donnent la même tendance. Cependant, le maximum de pression est sous-estimé.

L'évolution des efforts, calculés dans le repère $(X, Y, Z)$, système de coordonnées fixé au modèle, en fonction de l'angle de dérapage $\beta$, est donnée dans les figures 12. La traînée, voir figure 12a, augmente en fonction de l'angle $\beta$ jusqu'à $\beta=20^{\circ}$. Après cet angle, la traînée diminue. Ce comportement est typique d'un modèle à culot droit [1]. Ce comportement est trouvé par les deux types de simulations mais avec des résultats différents. Pour les simulations avec le modèle EASM, la traînée 


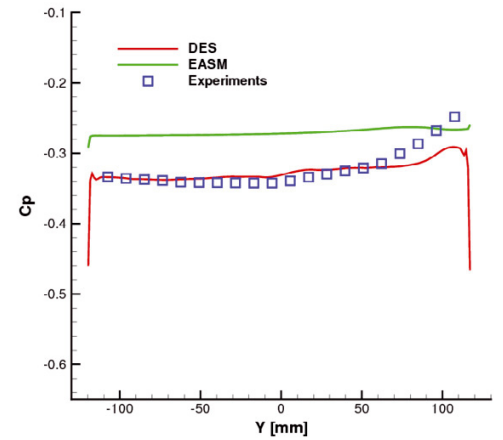

(a)

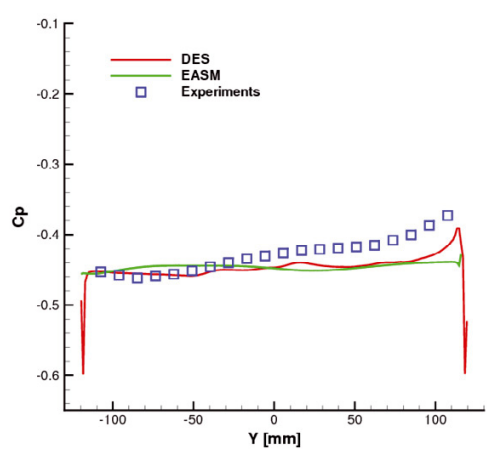

(b)

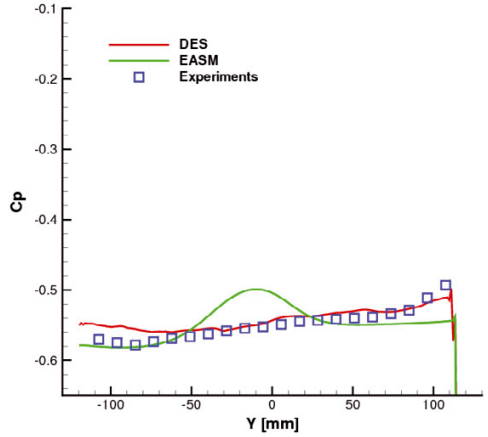

(c)

Fig. 11. Coefficient de pression sur la courbe (Pt), (a) $\beta=10^{\circ}$, (b) $\beta=20^{\circ}$, (c) $\beta=30^{\circ}$.

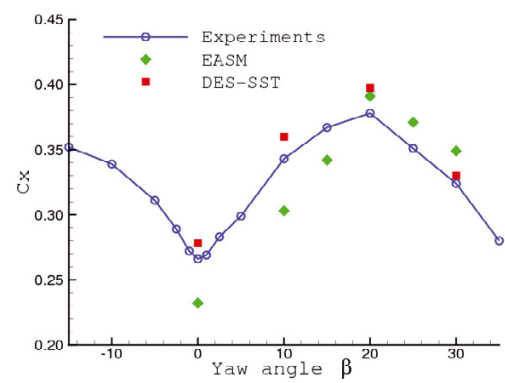

(a)

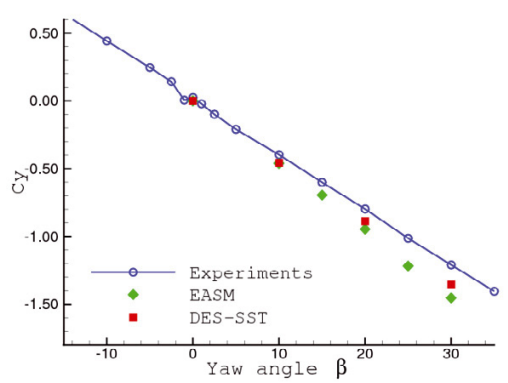

(b)

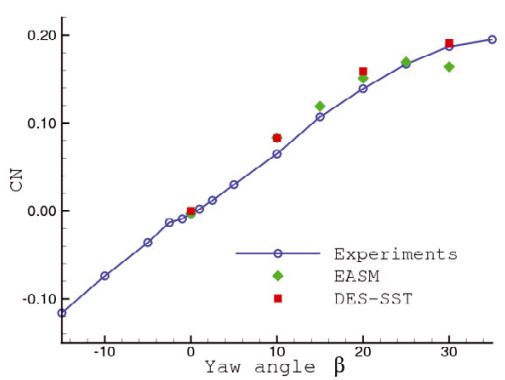

(c)

Fig. 12. Efforts dans le repère $(X, Y, Z)$ en fonction de l'angle de dérapage $\beta$, (a) coefficient de traînée Cx, (b) coefficient de la force latérale $\mathrm{Cy}$, (c) coefficient du moment de dérapage $\mathrm{CN}$.

est sous-estimée jusqu'à $\beta=20^{\circ}$. Passé cet angle de dérapage, la traînée est surestimée. Avec les simulations DES, la traînée est toujours surestimée par rapport aux résultats expérimentaux. On note que la valeur obtenue par les deux modélisations sont quasiment identique pour l'angle de dérapage $\beta=20^{\circ}$. Cependant, les résultats obtenus avec les simulations DES sont en meilleur accord avec les données expérimentales. La force latérale, voir figure $12 \mathrm{~b}$, montre une évolution linéaire en fonction de l'angle de dérapage $\beta$. Ce comportement est également reproduit par les deux simulations. Seule, la pente est surestimée. L'évolution du moment de dérapage en fonction de l'angle $\beta$ est présentée sur la figure 12c. Ce coefficient croît linéairement en fonction de l'angle de dérapage jusqu'à $\beta=25^{\circ}$. Après cet angle, la pente de la courbe diminue. Pour les simulations avec le modèle EASM, la pente a chuté, ce qui fait que le moment est sous-estimé. Les linéarités de la force latérale et du moment de dérapage sont caractéristiques typiques des véhicules réels [1].

\section{Conclusions}

Cet article présente une utilisation des simulations DES pour prédire les effets d'un vent latéral sur un modèle simplifié de voiture. Ces calculs ont été réalisés à l'aide du solveur Navier-Stokes ISIS-CFD, code de calcul développé au sein de l'Équipe Modélisation Numérique du Laboratoire de Mécanique des Fluides. Des comparaisons avec des données expérimentales et des résultats numériques obtenus avec une modélisation de la turbulence sont présentées. On note que les résultats obtenus par les simulations DES sont en meilleur accord avec les données expérimentales, aussi bien pour la pression totale que les efforts et le moment de dérapage. Une amélioration notable des résultats avec une simulation DES est la prédiction de la pression au niveau du culot du modèle.

Les efforts calculés sur le corps en fonction de l'angle de dérapage montrent les mêmes caractéristiques typiques que celles observées sur une voiture réelle. Les calculs ont montré que, pour le côté sous le vent, un décollement, situé vers le haut de la partie verticale du côté, est présent dès que l'angle de dérapage atteint $10^{\circ}$. Un deuxième tourbillon est également présent, situé sur la partie basse du côté. Jusqu'à un angle de dérapage de $20^{\circ}$, ce tourbillon reste proche du corps. Passé cet angle, celui-ci est très éloigné.

Cet article montre que les simulations DES peuvent être utilisées dans les études d'aérodynamique de véhicules terrestres et que ce type de simulation apporte une réponse plus précise de l'écoulement.

\section{Références}

[1] W. Hucho, Aerodynamics of road vehicles, SAE International, 1998 
[2] S. Maddox, K. Squires, J. Forsythe, Detached-eddy simulation of the flow around the ground transportation system, in The Aerodynamics of Heavy Vehicles: Trucks, Buses, Trains, in: B. McCallen, Ross (ed.), Vol. 19, Springer, 2004

[3] H. Hemida, Large-Eddy Simulation of the Flow around Simplified High-Speed Trains under Side Wind Conditions, Ph.D. thesis, Chalmers University of Technology, 2006

[4] C. Baker, N. Humphreys, Assessment of the adequacy of various wind tunnel techniques to obtain aerodynamic data for ground vehicles in cross winds, Journal of Wind Engineering and Industrial Aerodynamics 60 (1996) 4968

[5] A. Ryan, R. Dominy, The aerodynamic forces induced on a passenger vehicle in response to a transient cross-wind gust at a relative incidence of $30^{\circ}$, SAE Technical Paper 980392, 1998

[6] E. Guilmineau, Numerical simulation of wakes behind a car model, In FISITA World Automotive Congress, Yokohama, Japan, 2006

[7] F. Chometon, A. Strzelecki, J. Laurent, E. Aïssaoui, Experimental analysis of unsteady wakes on a new simple car model, in Fifth International Colloquium on Bluff Body Aerodynamics and Applications, Ottawa, Ontario, Canada, 2004, pp. 545-548
[8] E. Guilmineau, F. Chometon, Effect of side wind on a simplified car model: Experimental and numerical analysis, Journal of Fluids Engineering 31 (2009)

[9] H. Jasak, H. Weller, A. Gosman, High resolution nvd differencing scheme for arbitrarily unstructured meshes, International Journal for Numerical Methods in Fluids 31 (1999) 431-449

[10] F. Menter, M. Kuntz, Adaptation of eddy-viscosity turbulence models to unsteady separated flow behind vehicles, in Conference on The Aerodynamics of Heavy Vehicles: Trucks, Busses and Trains, Asilomar, CA, 2002

[11] A. Travin, M. Shur, M. Strelets, P. Spalart, Detachededdy simulations past a circular cylinder, in Flow, Turbulence and Combustion, Kluwer Academic Publishers, 2000, Vol. 63, pp. 293-313

[12] F. Menter, Two-equation eddy viscosity turbulence models for engineering applications, AIAA Journal 32 (1994) $1299-1310$

[13] S. Krajnovic, L. Davidson, Flow around a simplified car - part 1: Large eddy simulation, Journal of Fluids Engineering 127 (2005) 907-918

[14] M. Gohlke, J. Beaudoin, M. Amielh, F. Anselmet, Experimental analysis of flow structures and forces on a 3d-bluff-body in constant cross-wind, Experiments in Fluids 43 (2007) 579-594 